\title{
Quantification of Solute Deuterium in Titanium Deuteride by Atom Probe Tomography with Both Laser Pulsing and High-Voltage Pulsing: Influence of the Global and Local Surface Electric Field
}

\author{
Y. H. Chang ${ }^{1}$, I. Mouton ${ }^{1,2}$, L. Stephenson ${ }^{1}$, M. Ashton ${ }^{1}$, G. K. Zhang ${ }^{3}$, A. Szczpaniak ${ }^{1}$, D. Ponge ${ }^{1}$, D. \\ Raabe $^{1}$ and B. Gault ${ }^{1,4}$ \\ 1. Max Planck Institute für Eisenforschung GmbH, Düsseldorf, Germany. \\ 2. Laboratoire d'Analyse Microstructurale des Matériaux, Service de Recherches Métallurgiques \\ Appliquées, DEN, CEA, Gif-sur-Yvette, France. \\ 3. Institute of Materials, China Academy of Engineering Physics, Jiangyou, China. \\ 4. Department of Materials, Royal School of Mines, Imperial College, London, UK. \\ * Corresponding author: b.gault@mpie.de,i.mouton@mpie.de
}

Atom probe tomography (APT) has been increasingly used to investigate hydrogen embrittlement in metals due to its unique capacity for direct imaging of $\mathrm{H}$ atoms interacting with microstructural features $[1,2]$. However, the quantitativeness of hydrogen measurements by APT is yet to be established in views of erroneous compositional measurements of bulk hydrides [3] and the influence of spurious hydrogen, e.g. residual gas inside the analysis chamber [4]. Here, by using a stable stoichiometric titanium deuteride TiD 2 (approx. 66 at.\% D) in lieu of a hydride to minimize the overlap with residual gas, we systematically investigated the performance of APT towards quantitative analysis of solute $\mathrm{H}$, here D, with both laser pulsing and HV pulsing modes in both the LEAP 5000XS and LEAP 5000XR atom probe systems. Cryogenic focused ion beam (cryo-FIB) technique is deployed to avoid undesired introduction of $\mathrm{H}$ from the environment during specimen preparation, and subsequent vacuum-cryotransfer prevents out-diffusion of D from the deuteride, by significantly lowering H/D diffusion rate.

The dependencies of D quantification on experimental conditions, such as pulse repetition rate or laser pulse energy, are interpreted with regards to the strength of the surface electrostatic field derived from the charge-state ratio (Fig. 1). With laser pulsing (Fig. 1a), the overall measured compositions vary remarkably with the evaporation conditions in both atom probe configurations. In the low field regime (high laser pulse energy), the D fractions are significantly lower than the nominal value in both instruments, and they increase with the surface electric field, following a similar trend. In the high field regime (low laser pulse energy), the measured D compositions in the LEAP 5000XS increase dramatically and reach up to 80 at. $\%$, while the $\mathrm{D}$ compositions measured in the LEAP 5000XR remain below the stoichiometry. In contrast, compositional analyses with $\mathrm{HV}$ pulsing in both atom probe systems appear nearly independent of the applied experimental parameters, although approx. 15-20 at. \% off the nominal composition (Fig. 1b). A special emphasis is placed on the local variations of the measured composition as a function of the local electric field across the specimen's surface. As shown in Fig 2a, the microscopic chemical composition analyzed with laser pulsing is inhomogeneous and varies greatly with the local surface field due to the asymmetric laser illumination and inhomogeneous crystallographic structure of the tip surface. But with HV pulsing (Fig. 2b), the locally measured composition is much more homogeneous across the tip surface except along the crystallographic features. Therefore, for future application in imaging the local H/D distribution within microstructural defects in metals by APT, HV pulsing is recommended since the composition measurement is independent of the analysis parameters and specimen geometry both globally and locally. Our investigations demonstrate the challenges of quantitative analysis of solute deuterium by APT but 
nevertheless provide insight to achieving the best possible experimental protocol [5].

References:

[1] J Takahashi, K Kawakami and T Tarui, Scr. Mater. 67 (2012), p. 213.

[2] YS Chen, et al., Science (80) 355 (2017), p. 1196.

[3] Y Chang et al., Acta Mater. 150 (2018), p. 273.

[4] G Sundell, M Thuvander and HO Andrén, Ultramicroscopy 132 (2013), p. 285.

[5] The authors acknowledge financial support from the ERC-CoG-SHINE-771602. YC is grateful to the China Scholarship Council (CSC) for funding of PhD scholarship.

(a)

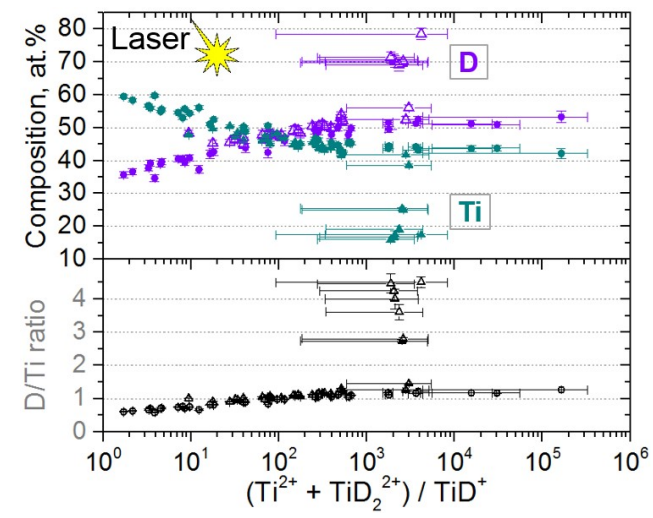

(b)

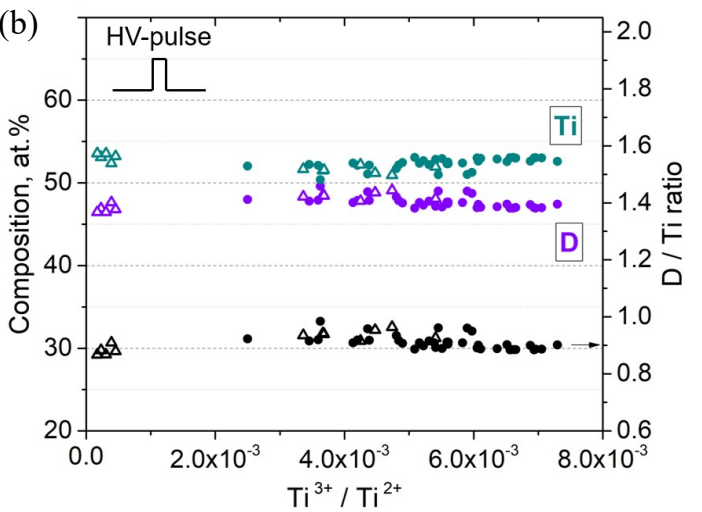

Figure 1. Measured compositions of $\mathrm{D}$ and $\mathrm{Ti}$, and the $\mathrm{D} / \mathrm{Ti}$ ratio as a function of global surface electric field for the measurements with laser pulsing (a) and HV pulsing (b). Data obtained from the LEAP 5000XR are labeled in circles $(\bullet)$ and from the LEAP 5000XS in upper triangles $(\Delta)$.

(a)

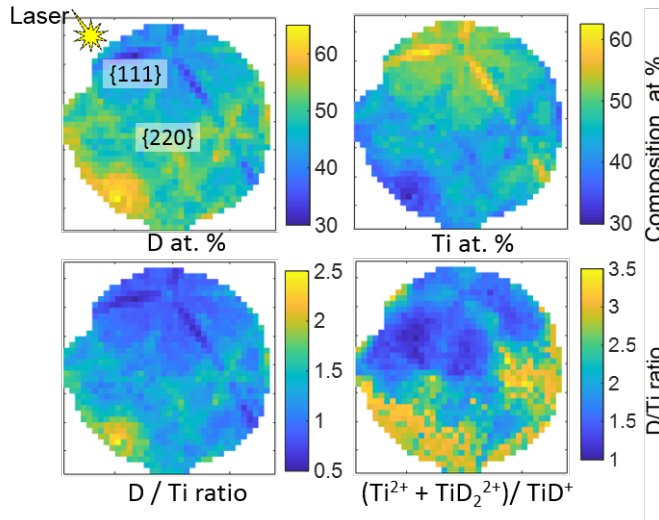

(b)

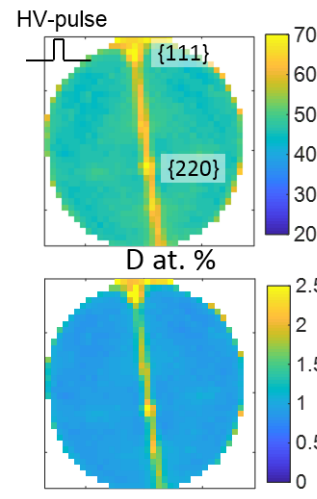

D / Ti ratio

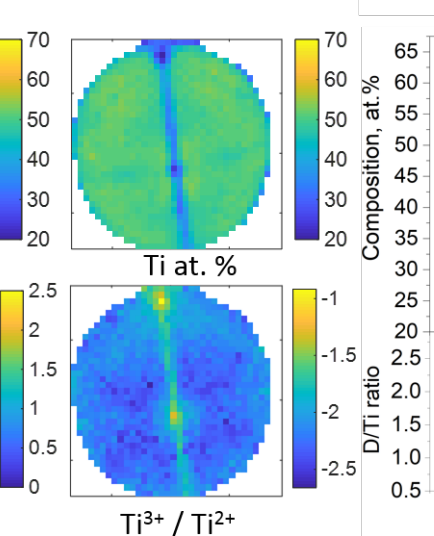

$\mathrm{Ti}^{3+} / \mathrm{Ti}^{2+}$

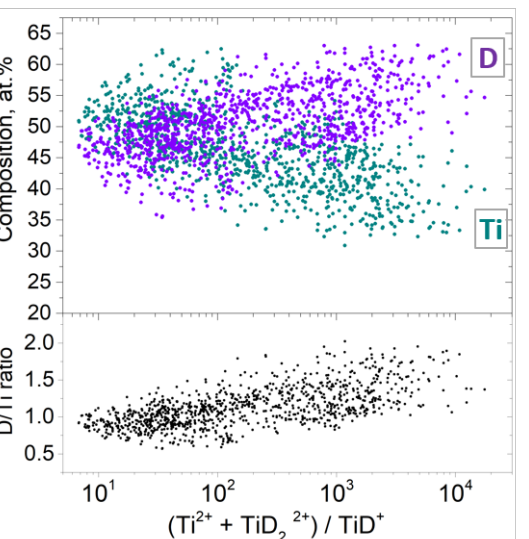

$\left(\mathrm{Ti}^{2+}+\mathrm{TiD}_{2}{ }^{2+}\right) / \mathrm{TiD}^{+}$

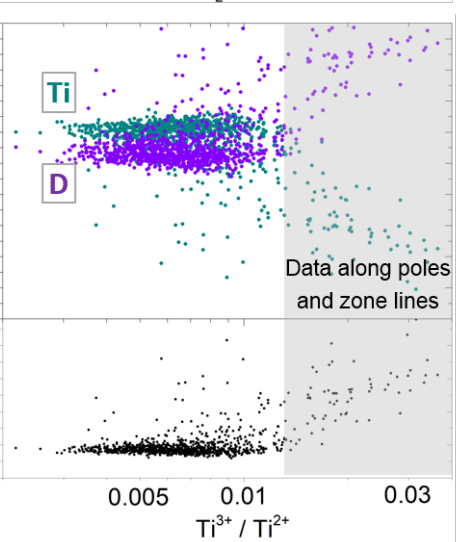

Figure 2. Measured compositions of $\mathrm{D}$ and $\mathrm{Ti}$, and the $\mathrm{D} / \mathrm{Ti}$ ratio as a function of local surface electric field for the measurements with laser pulsing (a) and high-voltage pulsing (b). 